\title{
MYG1 promotes proliferation and inhibits autophagy in lung adenocarcinoma cells via the AMPK/mTOR complex 1 signaling pathway
}

\author{
XIAODAN HAN ${ }^{1}$, AILI LI ${ }^{1}$, WEI WANG ${ }^{1}$, LONGXIA DU ${ }^{1}$, CHEN WANG $^{1}$ and GUOJIN HUANG ${ }^{1,2}$ \\ ${ }^{1}$ Laboratory of Respiratory Diseases, The Affiliated Hospital of Guilin Medical University; ${ }^{2}$ Guangxi Key Laboratory of \\ Molecular Medicine in Liver Injury and Repair, Guilin Medical University, Guilin, Guangxi 541001, P.R. China
}

Received August 16, 2020; Accepted January 14, 2021

DOI: 10.3892/ol.2021.12595

\begin{abstract}
Melanocyte proliferating gene 1 (MYG1) is an exonuclease that participates in RNA processing and is required for normal mitochondrial function. However, its role in tumorigenesis remains unknown. The present study aimed to investigate the role of MYG1 and its underlying mechanisms in human lung adenocarcinoma (LUAD). The expression levels of MYG1 in tumor tissues of patients with LUAD were obtained from public cancer databases and analyzed using the UALCAN online software. The association between MYG1 expression levels and the prognosis of patients with LUAD was analyzed using the Kaplan-Meier plotter. In addition, the role of MYG1 in the LUAD A549 and H1993 cell lines was determined by knocking down MYG1 expression with a specific small interfering RNA or by overexpressing it with a MYG1-containing plasmid. The results demonstrated that MYG1 expression levels were upregulated in LUAD tissues compared with those in normal lung tissues from healthy subjects, and high MYG1 expression levels were associated with an unfavorable prognosis. MYG1 promoted the proliferation, migration and invasion of A549 and H1993 cells. In addition, MYG1 inhibited autophagy via the AMP-activated protein kinase/mTOR complex 1 signaling pathway. Collectively, the present results suggested that MYG1 may serve an oncogenic role in LUAD and may be a potential therapeutic target for LUAD.
\end{abstract}

\section{Introduction}

Lung cancer is the most commonly diagnosed type of cancer and the leading cause of cancer-related mortality worldwide (1). Non-small cell lung cancer accounted for $85 \%$ of

Correspondence to: Dr Guojin Huang, Laboratory of Respiratory Diseases, The Affiliated Hospital of Guilin Medical University, 15 Lequn Road, Guilin, Guangxi 541001, P.R. China

E-mail: hgjj@163.com

Key words: melanocyte proliferating gene 1, lung adenocarcinoma, proliferation, autophagy, A549, H1993 lung cancer cases and $>85 \%$ of lung cancer-related mortalities in 2006 (2). As the most common type of non-small cell lung cancer, lung adenocarcinoma (LUAD) accounted for $~ 50 \%$ of non-small cell lung cancer cases in 2006 (2). Understanding of the molecular mechanisms of LUAD has improved during the last few decades, and thus, the treatments for LUAD, such as targeted therapy and immunotherapy, have been applied in clinical management and improved patient progression-free or overall survival (3). However, due to drug resistance and the need of personized medicine, it is crucial to discover novel therapeutic target molecules involved in LUAD (3).

mTOR is a serine/threonine protein kinase that coordinates cell proliferation and metabolism in response to nutrients and growth factors, and serves a central role in regulating numerous fundamental cell processes, such as protein synthesis and autophagy (4). mTOR forms the catalytic subunit of two distinct protein complexes, known as mTOR complex (mTORC) 1 and 2. mTORC1 comprises three core components, namely mTOR, regulatory protein associated with mTOR and mammalian lethal with Sec13 protein 8 , as well as two inhibitory subunits, proline-rich Akt substrate of $40 \mathrm{kDa}$ and DEP domain-containing mTOR interacting protein (4). mTORC1 functions as a downstream effector for multiple oncogenic pathways, including the PI3K/AKT and the Ras/Raf/MEK/MAPK pathways, resulting in mTORC1 hyperactivation in numerous types of human cancer, such as LUAD and colorectal carcinoma (4). Mammalian AMP-activated protein kinase (AMPK), which is composed of catalytic subunits $\alpha$ and $\beta$, and a regulatory $\gamma$ subunit, is an energy sensor that is sensitive to the cellular levels of ATP (5). AMPK is phosphorylated to an active state following ATP depletion (5). In addition, AMPK acts upstream of mTORC1 and links mTORC1 to energy stresses (5).

The human melanocyte proliferating gene 1 (MYG1) is a 376-amino acid protein that harbors a mitochondrial targeting sequence among amino acids 1-20 and a nuclear localization signal among amino acids 33-39; therefore, the protein is localized in the nucleus and the mitochondria (6). MYG1 is ubiquitously expressed in all human tissues, with the highest levels in the testis (6). A previous study has reported that the $-119 \mathrm{C} / \mathrm{G}$ polymorphism in the promoter of MYG1 is associated with vitiligo susceptibility, and an Arg4Gln 
polymorphism in the mitochondrial signal of MYG1 affects mitochondrial localization (7). Furthermore, MYG1 has been reported to act as a $3^{\prime}-5^{\prime}$ RNA exonuclease and to be involved in the maturation of ribosomal and messenger RNA in the mitochondria; thus, it is required for mitochondrial functions (8). However, the roles of MYG1 in tumorigenesis and the potential underlying mechanism remain unknown.

The present study aimed to examine the expression levels of MYG1 in LUAD tissues and analyze the association between MYG1 expression and the prognosis of patients with LUAD. In addition, the present study aimed to investigate the function of MYG1 and its underlying mechanism in A549 and H1993 LUAD cells.

\section{Materials and methods}

Antibodies, plasmids and small interfering (si)RNA. The antibody against MYG1 (cat. no. sc-393331) was purchased from Santa Cruz Biotechnology, Inc.. Antibodies against p62 (cat. no. 39749S), AMPKa (cat. no. 2603), phosphorylated (p)-AMPK $\alpha$ (Thr172) (cat. no. 2535S), p70 S6 kinase (S6K; cat. no. 9202), p-S6K (cat. no. 9204), and HRP-conjugated anti-mouse (cat. no. 7076) and anti-rabbit (cat. 7074) IgG antibodies were purchased from Cell Signaling Technology, Inc. The antibody against microtubule-associated proteins $1 \mathrm{~A} / 1 \mathrm{~B}$ light chain 3B (LC3B; cat. no. ab192890) was obtained from Abcam, and the antibody against GAPDH (cat. no. TA505454) was purchased from OriGene Technologies, Inc.. The dilutions of the antibodies used for western blotting were 1:500 for MYG1, 1:1,000 for LC3B, p62, AMPK $\alpha$, p-AMPK $\alpha$, S6K, p-S6K and GAPDH, and 1:3,000 for HRP-conjugated anti-mouse and anti-rabbit IgG antibodies.

The cDNA of MYG1 tagged with FLAG at the C-terminus was synthesized by ShineGene Bio-Technologies, Inc. and cloned into the pFlag-CMV-4 vector, which was kindly provided by Dr Xianqiong Zou (Guilin Medical University, Guilin, China). The empty pFlag-CMV-4 vector was used as the negative control. MYG1 siRNA (MYG1 siRNA-1, 5'-GGA CGCACAAUGGCACCUUTT-3'; MYG1 siRNA-2, 5'-GGU CUUUCACAGAGACCAUTT-3'; MYG1 siRNA-3, 5'-GCC CAGUUGCUGGGCACUATT-3') and the negative control siRNA (control siRNA, 5'-UUCUCCGAACGUGUCACG UTT-3') were synthesized by Shanghai GenePharma Co., Ltd. .

Cell culture and transfection. The A549 and H1993 LUAD cell lines were purchased from the Kunming Cell Bank of the Chinese Academy of Sciences. The cells were cultured in RPMI-1640 medium (Gibco; Thermo Fisher Scientific, Inc.) supplemented with 10\% FBS (Gibco; Thermo Fisher Scientific, Inc.) at $37^{\circ} \mathrm{C}$ in a humidified incubator with $5 \% \mathrm{CO}_{2}$.

A549 and H1993 LUAD cells $\left(1.5 \times 10^{5}\right)$ were seeded into 6-well plates and cultured overnight, and transfection was performed using Lipofectamine ${ }^{\circledR} 3000$ (Invitrogen; Thermo Fisher Scientific, Inc.) according to the manufacturer's instructions. The transfection reagent was incubated with siRNA or plasmids at room temperature for $15 \mathrm{~min}$ and added to the 6-well plates. A total of $2 \mu \mathrm{g}$ plasmids/well was used for overexpression experiments. MYG1 siRNA (75 pmol) and control siRNA (75 pmol) were used for RNA interference experiments. Cells were cultured at $37^{\circ} \mathrm{C}$ and collected at $48 \mathrm{~h}$ post-transfection.
Colony formation assay. Transfected A549 and H1993 cells were seeded into 6-well plates in $2 \mathrm{ml}$ growth medium at a density of $1 \times 10^{3}$ cells/well and cultured in RPMI-1640 with $10 \% \mathrm{FBS}$ at $37^{\circ} \mathrm{C}$ in a humidified incubator with $5 \% \mathrm{CO}_{2}$ for 10-15 days. The culture medium was replaced every 2 days. The cells were washed three times with PBS and fixed with $4 \%$ neutral paraformaldehyde solution at room temperature for $30 \mathrm{~min}$, followed by another three washes with PBS. Subsequently, $2 \mathrm{ml} 0.5 \%$ crystal violet solution was added to each well and incubated at room temperature for $2 \mathrm{~h}$. The cells were washed three times with PBS. The plates were dried and scanned with Epson Perfection V370 Photo scanner (Seiko Epson Corporation). Cell colonies visible by the naked eye were manually counted.

Western blotting. Western blot analysis was performed as previously described (9). The cells were washed three times with cold PBS and lysed with RIPA buffer (cat. no. R0020; Beijing Solarbio Science \& Technology Co., Ltd.) on ice for $40 \mathrm{~min}$. The lysate was centrifuged at $13,500 \mathrm{x}$ g for $15 \mathrm{~min}$ at $4^{\circ} \mathrm{C}$ to obtain the supernatant, in which the protein concentration was measured with a BCA Kit (Beyotime Institute of Biotechnology) according the manufacturer's instructions. Proteins $(30 \mathrm{mg} / \mathrm{lane})$ were separated with 10 or $15 \%$ sodium dodecyl sulfate-polyacrylamide gels and transferred on a PVDF membrane, which was blocked with $5 \%$ non-fat milk at room temperature for $2 \mathrm{~h}$. Subsequently, the membrane was incubated with the primary antibodies at $4^{\circ} \mathrm{C}$ overnight, washed with $1 \mathrm{X}$ TBST buffer, and incubated with the secondary antibody (HRP-conjugated anti-mouse or anti-rabbit IgG antibody) at room temperature for $1 \mathrm{~h}$. Blots were developed using the SuperSignal ${ }^{\mathrm{TM}}$ West Femto substrate (Bridgen Biotechnology Co., Ltd.). The optical density of the protein bands was semi-quantified with ImageJ $1.53 \mathrm{a}$ software (National Institutes of Health) and normalized to that of the loading control.

Invasion and migration assays. At $48 \mathrm{~h}$ post-transfection, A549 and H1993 cells ( $3 \times 10^{3}$ cells/well) were seeded into a 24-well Transwell insert (8- $\mu \mathrm{m}$ pore size; BD Biosciences) with or without Matrige $\mathrm{l}^{\circledR}$ according to the manufacturer's instructions at room temperature for $30 \mathrm{~min}$. FBS-free medium was added to the upper chamber, whereas medium supplemented with $20 \%$ FBS was added to the lower chamber. After incubation at $37^{\circ} \mathrm{C}$ with $5 \% \mathrm{CO}_{2}$ for $24 \mathrm{~h}$, non-invading or non-migrating cells were removed from the top wells with a cotton swab. The cells that had transgressed to the bottom of the membrane were fixed with $4 \%$ paraformaldehyde at room temperature for $15 \mathrm{~min}$ and stained with $0.2 \%$ crystal violet at $4^{\circ} \mathrm{C}$ overnight. Images of five randomly selected independent fields from each well were captured under an Olympus TH4-200 phase-contrast microscope (x200 magnification; Olympus Corporation). The cells in each image were quantified with ImageJ $1.53 \mathrm{a}$ software, and the results were presented as a percentage of the control group.

A wound healing assay was conducted to determine cell migration. The cells were cultured to confluent monolayers in 6-well plates. The monolayers were scratched with a $10-\mu 1$ pipette tip and washed twice with 1X PBS. A549 and H1993 cells were cultured for 24 or $72 \mathrm{~h}$, respectively, and images 
were captured under an Olympus TH4-200 phase-contrast microscope (x200 magnification). The scratch area was measured with ImageJ 1.53a software. The quantitative results were calculated as described previously (10) and presented as wound closure percentage relative to the control group.

ATP assay. The ATP assay kit (cat. no. S0026) was purchased from Beyotime Institute of Biotechnology and performed according to manufacturer's instructions as previously described (11). Briefly, the transfected A549 and H1993 cells $\left(5 \times 10^{5}\right)$ were washed three times with cold PBS and lysed with ATP detection lysis buffer on ice for $40 \mathrm{~min}$; the lysate was centrifuged at $13,500 \mathrm{x}$ g for $15 \mathrm{~min}$ at $4^{\circ} \mathrm{C}$ to obtain the supernatant, and $20 \mu \mathrm{l}$ of the supernatant was mixed with $100 \mu \mathrm{l}$ substrate solution and incubated at room temperature for $10 \mathrm{sec}$. The luminescence was measured at $520 \mathrm{~nm}$ using a Synergy HTX Multi-Mode microplate reader (BioTek Instruments, Inc.).

MYG1 expression in LUAD and prognostic analysis. MYG1 expression in The Cancer Genome Atlas (TCGA) LUAD and normal tissue samples, as well as at different tumor stages were analyzed using the UALCAN online software (http://ualcan.path.uab.edu/) (12). The survival probability of patients with LUAD was analyzed using the Kaplan-Meier plotter (http://kmplot.com) (13). A log-rank test was used to assess statistical differences in survival probability, and the cutoff values of MYG1 expression were automatically selected by the software as follows: 527 transcripts per million (TPM) for overall survival (OS), 551 TPM for first progression (FP) and 781 TPM for post-progression survival (PPS).

Gene set enrichment analysis (GSEA). TCGA LUAD gene expression dataset was downloaded from cBioportal (www. cbioportal.org). LUAD samples were classified into high- and low-MYG1 expression groups using the median value of MYG1 expression as cutoff. GSEA was performed using GSEA 4.0.3 (http://www.broad.mit.edu/gsea/) with the pre-defined hallmark gene sets, each containing $>4,000$ genes $(14,15)$. The default settings were used, and the thresholds for significance were determined by permutation analysis $(1,000$ permutations). A gene set was considered significantly enriched when the false discovery rate was $<0.25$.

Analysis of the associations between MYG1 expression levels and the clinicopathological characteristics. The clinicopathological data of the patients in TCGA LUAD cohort was downloaded from http://www.cbioportal.org/datasets. The stages of LUAD were determined according to American Joint Committee on Cancer (AJCC) Staging (3rd-7th edition) (16). The patients from TCGA LUAD dataset were separated into a MYG1 high- and low-expression group using the median value of MYG1 expression as a cutoff, and the data were analyzed using the $\chi^{2}$ test.

Statistical analysis. All experiments were conducted $\geq 3$ times independently, and the data are presented as the mean $\pm \mathrm{SD}$. Statistical analyses were performed using GraphPad Prism 8 (GraphPad Software, Inc.) and SPSS version 11.0 (SPSS, Inc.). Comparisons between two groups were conducted using unpaired Student's t-test, and comparisons among multiple groups were performed using one-way ANOVA followed by Tukey's post hoc test. The data from TCGA LUAD dataset were analyzed using the $\chi^{2}$ test. $\mathrm{P}<0.05$ was considered to indicate a statistically significant difference.

\section{Results}

MYG1 expression levels are upregulated in LUAD. The expression levels of MYG1 in LUAD were determined by analyzing TCGA LUAD data using UALCAN (12). Compared with those in normal lung tissues, MYG1 expression levels were significantly higher in LUAD tissues (Fig. 1A). The expression levels of MYG1 at clinical stages 1-4 (stage 1, $\mathrm{n}=277$; stage $2, \mathrm{n}=125$; stage $3, \mathrm{n}=85$; and stage $4, \mathrm{n}=28$ ) were significantly upregulated compared with those in normal lung tissues ( $\mathrm{n}=59)$ (Fig. 1B). Additionally, the expression levels of MYG1 at stage 3 were significantly higher compared with those at stage 1 (Fig. 1B), whereas no significant differences were identified between any other stages of LUAD.

High expression levels of MYG1 are associated with an unfavorable prognosis in patients with LUAD. To identify the associations between MYG1 expression levels and the clinicopathological characteristics of patients with LUAD, the patients from TCGA LUAD dataset were separated into a MYG1 high- and low-expression group using the median value of MYG1 expression as a cutoff, and the data were analyzed using the $\chi^{2}$ test. The results demonstrated that MYG1 expression levels were significantly associated with the $\mathrm{N}$ stage (Table I).

To investigate the potential value of MYG1 in patient prognosis, the association between MYG1 expression levels and the survival of patients with LUAD was analyzed using the Kaplan-Meier plotter. The results demonstrated that high expression of MYG1 was associated with a poor survival rate of OS and PPS, but not associated with FP (Fig. 2). However, multivariate analysis indicated that MYG1 was not an independent prognostic factor for OS, FP or PPS (Table II).

MYG1 promotes the proliferation of LUAD cells. To study the function of MYG1 in LUAD, three specific siRNA molecules targeting MYG1 were synthesized; MYG1 siRNA-1 was selected for subsequent experiments as it exerted the strongest inhibitory effect on MYG1 protein expression (Fig. 3A).

Cell proliferation and colony formation were determined following overexpression or knockdown of MYG1 in A549 and H1993 cells. The results demonstrated that MYG1 overexpression promoted cell proliferation and colony formation compared with those in the empty vector-transfected cells, whereas knockdown of MYG1 exerted the opposite effects (Fig. 3B and C).

MYG1 promotes the migration and invasion of LUAD cells. To investigate whether MYG1 was involved in the migration and invasion of A549 and H1993 cells, the cells were transfected with MYG1 overexpression plasmids and an empty vector, or with MYG1 siRNA and control siRNA. Subsequently, Transwell and wound healing assays were performed. The results of the Transwell assays revealed that overexpression 

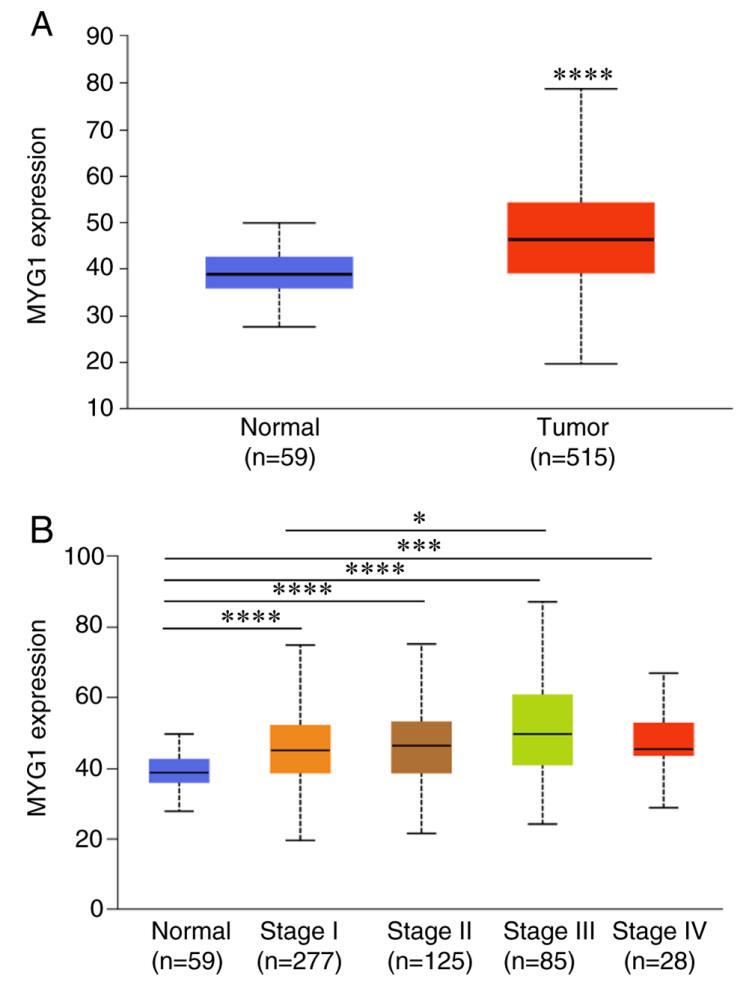

Figure 1. MYG1 is upregulated in LUAD compared with normal lung tissues. (A) MYG1 expression levels in LUAD $(n=515)$ and normal lung $(n=59)$ tissues from TCGA LUAD data were analyzed using UALCAN. MYG1 expression levels were significantly higher in LUAD compared with those in normal tissues. (B) MYG1 expression levels in LUAD tissue from patients at different American Joint Committee on Cancer stages and in normal lung tissues from TCGA were analyzed using UALCAN. MYG1 expression levels in all stages of LUAD were significantly higher compared with those in normal tissues; in addition, the expression levels of MYG1 at stage III were significantly higher compared with those at stage I. ${ }^{*} \mathrm{P}<0.05,{ }^{* * *} \mathrm{P}<0.001$ and ${ }^{* * * * *} \mathrm{P}<0.0001$. LUAD, lung adenocarcinoma; MYG1, melanocyte proliferating gene 1; TCGA, The Cancer Genome Atlas.

of MYG1 significantly increased the number of the cells that crossed the membrane compared with those in the control group, whereas the opposite results were obtained in the MYG1 knockdown group (Fig. 4A and B). The wound healing assay demonstrated that, compared with the corresponding control groups, overexpression of MYG1 increased cell migration, and MYG1 knockdown exerted the opposite effect (Fig. 4C and D). Collectively, the Transwell and wound healing assay results suggested that MYG1 promoted cell migration and invasion.

MYG1 inhibits autophagy in LUAD cells. Autophagy is a lysosomal degradation process that serves a dual role in cancer (17). Since LC3B conversion (LC3-I to LC3-II) and lysosomal degradation of LC3-II reflect the progression of autophagy, and the p62 protein is degraded by autophagy (18), LC3B and p62 are considered to be markers of autophagy. To identify the potential effects of MYG1 on autophagy, MYG1 was overexpressed or knocked down in A549 and H1993 cells. The expression levels of the autophagic markers p62 and LC3-I/II were detected by western blot analysis $48 \mathrm{~h}$ post-transfection. The results demonstrated that overexpression of MYG1 led to an increase in p62 protein expression levels, as well as a decrease in the ratio of LC3B-II/LC3B-I (Fig. 5), indicating reduced autophagy compared with the empty vector group.
By contrast, knockdown of MYG1 resulted in a decrease in p62 protein expression levels and an increase in the ratio of LC3B-II/LC3B-I (Fig. 5), suggesting enhanced autophagy compared with the control siRNA group. Taken together, these results suggested that MYG1 inhibited autophagy.

Knockdown of MYGl enhances the phosphorylation of $A M P K \alpha$ and suppresses mTORCl activity. To identify the underlying mechanisms via which MYG1 mediates the aforementioned cancerous features, TCGA LUAD gene expression data were analyzed using GSEA. The results demonstrated that the 'HALLMARK_OXIDATIVE_PHOSPHORYLATION' and 'HALLMARK_MTORC1_SIGNALING' gene sets were enriched with high expression of MYG1 (Fig. 6A and B), indicating that MYG1 may be associated with oxidative phosphorylation and mTORC1. To confirm the association between MYG1 expression and oxidative phosphorylation, the levels of the product of oxidative phosphorylation, cellular ATP, were measured after MYG1 knockdown and overexpression. The results demonstrated that the levels of cellular ATP were significantly decreased following knockdown of MYG1, but were significantly increased following MYG1 overexpression compared with those in the corresponding control groups (Fig. 6C).

AMPK is a cellular energy sensor, which is phosphorylated and activated during cellular ATP depletion (5). Therefore, phosphorylation of AMPK $\alpha$ was determined in A549 and H1993 cells following MYG1 knockdown and overexpression. The results demonstrated that knockdown of MYG1 enhanced the levels of AMPK $\alpha$ phosphorylation, whereas MYG1 overexpression decreased the levels of AMPK $\alpha$ phosphorylation compared with those in the corresponding control groups (Fig. 6D). mTOR is a serine/threonine kinase that forms mTORC1, which is downstream of AMPK and negatively regulates autophagy (4). To determine mTORC1 activity following MYG1 knockdown or overexpression, the phosphorylation of the mTORC1 substrate p70S6K was detected by western blot analysis. The results demonstrated that the phosphorylation levels of p70S6K decreased following MYG1 knockdown, but increased following MYG1 overexpression compared with those in the corresponding control groups (Fig. 6D). Collectively, these results suggested that MYG1 was positively associated with cellular ATP production, and that high levels of MYG1 expression may promote mTORC1 activity via AMPK.

\section{Discussion}

MYG1 is ubiquitously expressed in healthy human tissues and is localized in the nucleus and mitochondria (6). MYG1 is an exonuclease that participates in RNA processing and is required for mitochondrial functionality (8). Mitochondria are key factors in tumorigenesis due to their roles in energy production, regulation of cell signaling and cell death (19). To the best of our knowledge, no published studies are currently available on the function and clinical significance of MYG1 in tumors, and thus, the present study aimed to investigate the potential role of MYG1 in LUAD.

To examine the role of MYG1 in tumorigenesis, the present study first compared the expression levels of MYG1 in LUAD 
Table I. Associations between MYG1 expression levels and the clinicopathological characteristics of patients in The Cancer Genome Atlas lung adenocarcinoma dataset.

\begin{tabular}{|c|c|c|c|c|c|}
\hline \multirow[b]{2}{*}{ Characteristics } & \multirow[b]{2}{*}{ Total, $\mathrm{n}$} & \multicolumn{2}{|c|}{ MYG1 expression, $\mathrm{n}$} & \multirow[b]{2}{*}{$\chi^{2}$} & \multirow[b]{2}{*}{ P-value } \\
\hline & & Low & High & & \\
\hline \multicolumn{6}{|l|}{ Age, years } \\
\hline$<60$ & 138 & 63 & 75 & 0.9602 & 0.3271 \\
\hline$\geq 60$ & 364 & 184 & 180 & & \\
\hline \multicolumn{6}{|l|}{ Sex } \\
\hline Female & 277 & 132 & 145 & 1.2080 & 0.2717 \\
\hline Male & 240 & 126 & 114 & & \\
\hline \multicolumn{6}{|l|}{ T stage } \\
\hline $\mathrm{T} 1$ & 168 & 84 & 84 & 0.0237 & 0.8776 \\
\hline $\mathrm{T} 2 / 3 / 4$ & 345 & 170 & 175 & & \\
\hline $\mathrm{T} 1 / 2$ & 449 & 227 & 222 & 1.2710 & 0.2596 \\
\hline $\mathrm{T} 3 / 4$ & 65 & 28 & 37 & & \\
\hline \multicolumn{6}{|l|}{$\mathrm{N}$ stage } \\
\hline No & 380 & 168 & 212 & 19.0900 & $<0.0001^{\mathrm{a}}$ \\
\hline $\mathrm{N} 1 / 2 / 3$ & 126 & 84 & 42 & & \\
\hline \multicolumn{6}{|l|}{ M stage } \\
\hline M0 & 354 & 171 & 183 & 0.1276 & 0.7209 \\
\hline M1 & 25 & 13 & 12 & & \\
\hline \multicolumn{6}{|l|}{ Clinical stage } \\
\hline Stage I & 278 & 141 & 137 & 0.0129 & 0.9095 \\
\hline Stage II/III/IV & 223 & 117 & 116 & & \\
\hline Stage I/ II & 399 & 204 & 195 & 0.5126 & 0.4740 \\
\hline Stage III/IV & 110 & 52 & 58 & & \\
\hline \multicolumn{6}{|c|}{ Predicted DLCO, \% } \\
\hline$<80$ & 124 & 64 & 60 & 1.4980 & 0.2210 \\
\hline$\geq 80$ & 75 & 32 & 43 & & \\
\hline \multicolumn{6}{|c|}{ Bronchodilator FEV1, \% } \\
\hline$<80$ & 50 & 20 & 30 & 0.0305 & 0.8615 \\
\hline$\geq 80$ & 77 & 32 & 45 & & \\
\hline \multicolumn{6}{|c|}{ Location in lung parenchyma } \\
\hline Central & 64 & 31 & 33 & 0.2123 & 0.6450 \\
\hline Peripheral & 127 & 66 & 61 & & \\
\hline \multicolumn{6}{|l|}{ Tumor status } \\
\hline Absent & 311 & 160 & 151 & 0.1006 & 0.7511 \\
\hline Present & 109 & 58 & 51 & & \\
\hline \multicolumn{6}{|c|}{ Smoking history indicator } \\
\hline $1 / 2$ & 196 & 94 & 102 & 0.5357 & 0.4642 \\
\hline $3 / 4 / 5$ & 306 & 157 & 149 & & \\
\hline \multicolumn{6}{|c|}{ Packs of cigarettes per year, $n$} \\
\hline$<40$ & 175 & 83 & 92 & 0.4116 & 0.5212 \\
\hline$\geq 40$ & 177 & 90 & 87 & & \\
\hline
\end{tabular}

${ }^{a} \mathrm{P}<0.0001$. MYG1, melanocyte proliferating gene 1; DLCO, diffusing capacity for carbon monoxide; FEV, forced expiratory volume.

tissues with those in healthy tissue using the data of TCGA LUAD cohort. The results demonstrated that MYG1 expression levels were significantly upregulated in LUAD compared with those in the normal tissues. However, MYG1 expression levels did not change significantly from LUAD stage 1 to stage 4, indicating that MYG1 upregulation occurs early during the tumorigenesis 
Table II. Cox regression multivariate analysis of MYG1 on overall survival, first progression and post-progression survival of patients with lung adenocarcinoma.

A, Overall survival

\begin{tabular}{lcll}
\hline Variable & Hazard ratio & $95 \% \mathrm{CI}$ & P-value \\
\hline Sex & 1.50 & $0.83-2.70$ & 0.1824 \\
Stage & 2.52 & $0.47-13.56$ & 0.2812 \\
AJCC stage T & 2.31 & $1.13-4.72$ & $0.0217^{\mathrm{a}}$ \\
AJCC stage N & 1.44 & $0.26-8$ & 0.6800 \\
Smoking history & 0.91 & $0.42-2$ & 0.8207 \\
MYG1 & 1.57 & $0.84-2.92$ & 0.1536 \\
\hline
\end{tabular}

B, First progression

\begin{tabular}{lccc}
\hline Variable & Hazard ratio & $95 \% \mathrm{CI}$ & P-value \\
\hline Sex & 1.25 & $0.65-2.42$ & 0.5035 \\
Stage & 1.02 & $0.1-9.95$ & 0.9855 \\
AJCC stage T & 3.03 & $1.25-7.36$ & $0.0142^{\mathrm{a}}$ \\
AJCC stage N & 2.98 & $0.31-28.31$ & 0.3416 \\
Smoking history & 1.39 & $0.66-2.96$ & 0.3867 \\
MYG1 & 1.36 & $0.66-2.81$ & 0.4053 \\
\hline
\end{tabular}

C, Post-progression survival

\begin{tabular}{lccr}
\hline Variable & Hazard ratio & $95 \%$ CI & P-value \\
\hline Sex & 1.26 & $0.57-2.81$ & 0.5715 \\
Stage & 0.73 & $0.06-8.27$ & 0.7984 \\
AJCC stage T & 1.94 & $0.58-6.45$ & 0.2796 \\
AJCC stage N & 2.55 & $0.21-30.97$ & 0.4615 \\
Smoking history & 1.37 & $0.44-4.24$ & 0.5852 \\
MYG1 & 1.89 & $0.75-4.78$ & 0.1795
\end{tabular}

${ }^{\mathrm{a}} \mathrm{P}<0.05$. MYG1, melanocyte proliferating gene 1; AJCC, American Joint Committee on Cancer.

A

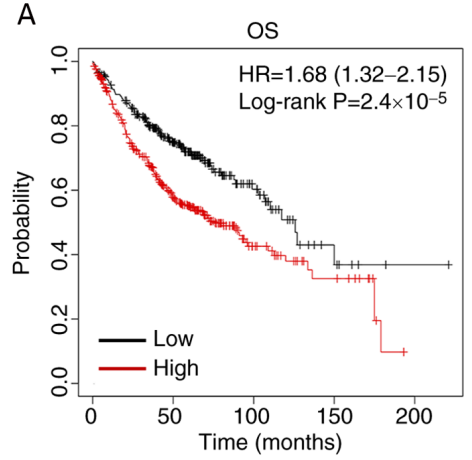

Number at risk

$\begin{array}{lllll}\text { Low } 316 & 169 & 36 & 7 & 1 \\ \text { High } 403 & 178 & 33 & 12 & 0\end{array}$

B

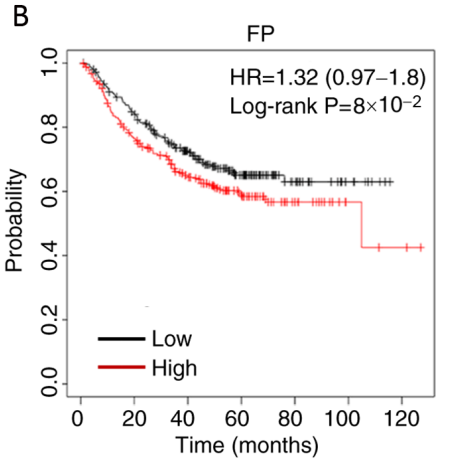

Number at risk

Low $248203 \quad 144 \quad 87 \quad 27 \quad 9 \quad 0$

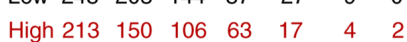

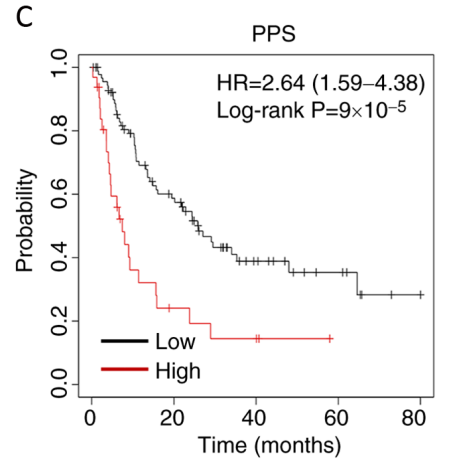

Number at risk

$\begin{array}{ccccc}\text { Low } 93 & 44 & 15 & 7 & 1 \\ \text { High } 32 & 5 & 3 & 0 & 0\end{array}$

Figure 2. High expression of MYG1 is associated with unfavorable OS and PPS of patients with lung adenocarcinoma. (A) High expression levels of MYG1 were associated with unfavorable OS (cut-off value, 527; HR=1.68; 95\% CI, 1.32-2.15; log-rank P=2.4x10 ${ }^{-5}$ ). (B) MYG1 was not associated with FP (cut-off value, 551; $\mathrm{HR}=1.32 ; 95 \% \mathrm{CI}, 0.97-1.8$; log-rank $\mathrm{P}=8.0 \times 10^{-2}$ ). (C) High expression levels of MYG1 were associated with unfavorable PPS (cut-off value, 781; $\mathrm{HR}=2.64$; $95 \% \mathrm{CI}, 1.59-4.38$; log-rank $\mathrm{P}=9.0 \times 10^{-5}$ ). Patients were separated using an auto-select best cutoff. OS, overall survival; FP, first progression; MYG1, melanocyte proliferating gene 1; HR, hazard ratio; PPS, post-progression survival. 
A

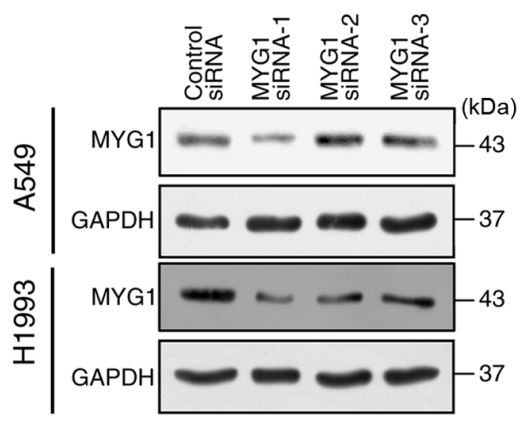

B

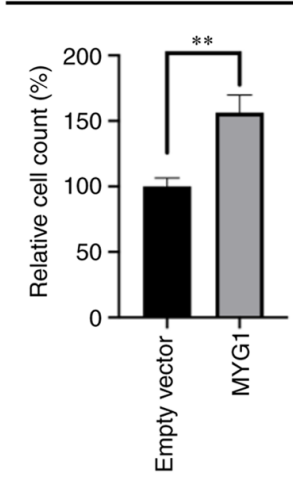

A549

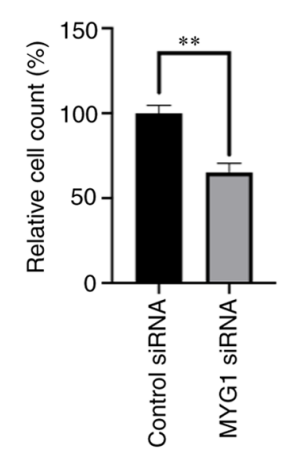

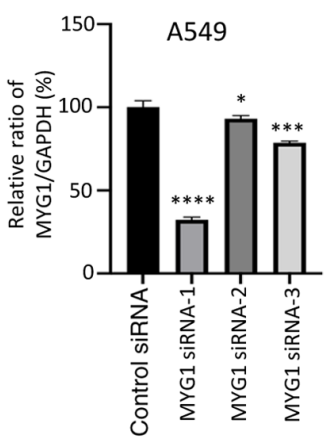

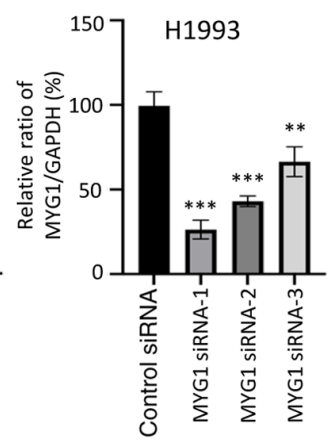

H1993

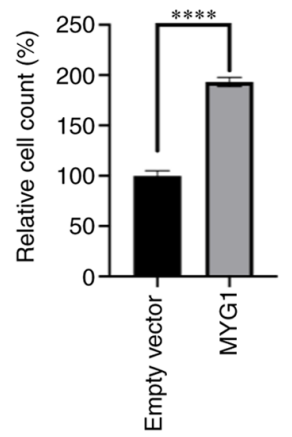

C

A549
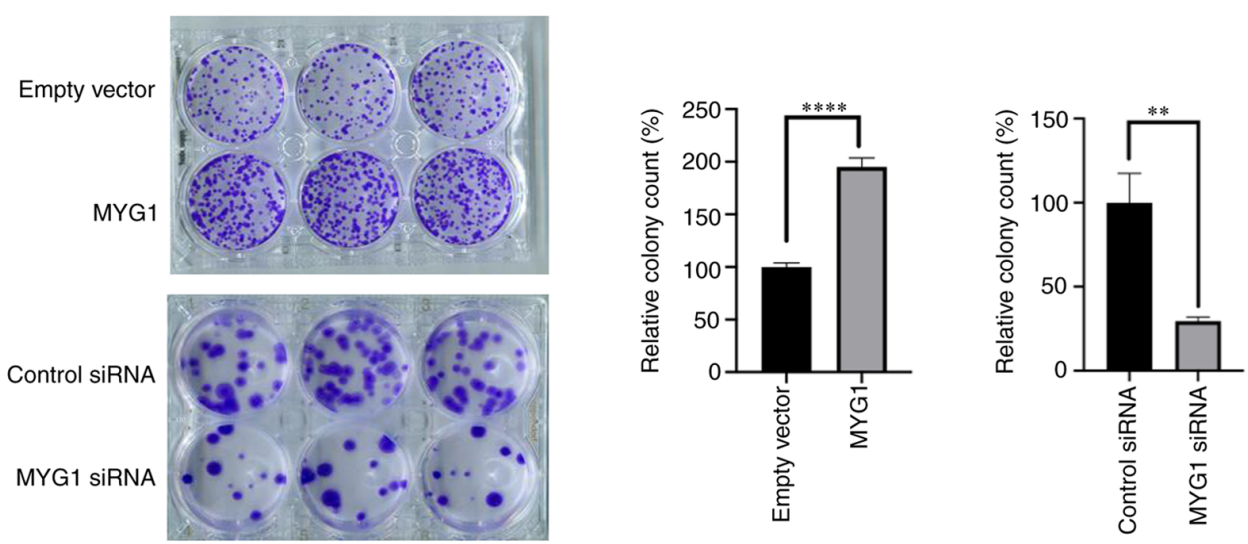

H1993
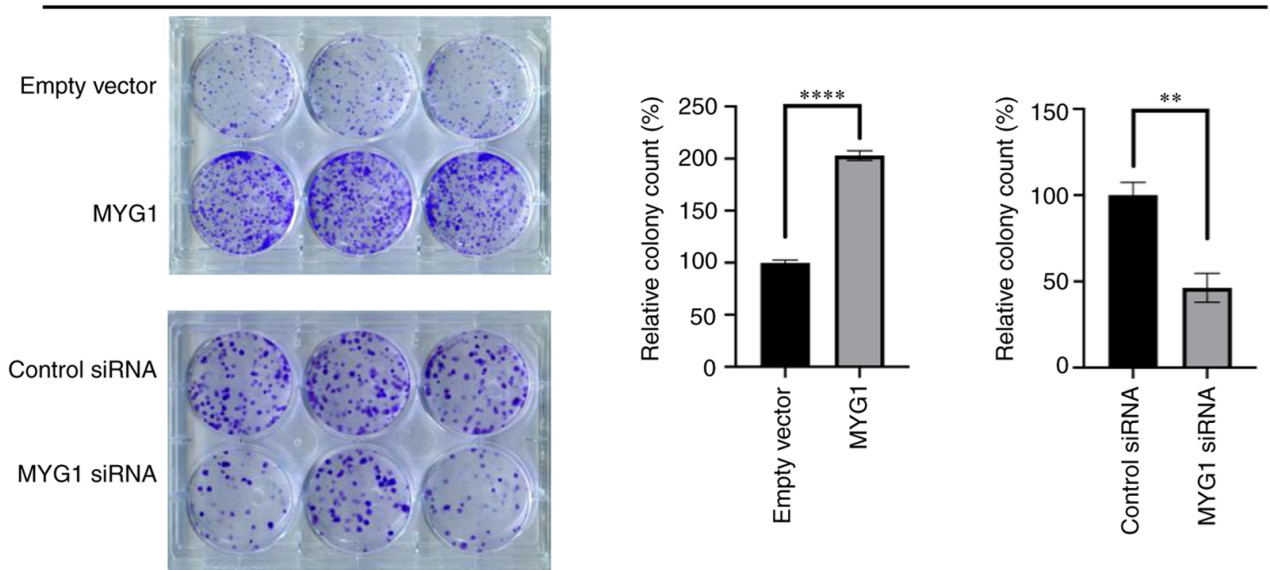

Figure 3. MYG1 promotes cell proliferation and colony formation of lung adenocarcinoma cells. (A) The effects of MYG1 siRNA on its protein expression (B) MYG1 promotes cell proliferation. A549 and H1993 cells were transfected with MYG1 plasmids or siRNA for $48 \mathrm{~h}$, and proliferation was determined by counting the number of cells. (C) MYG1 promotes colony formation in A549 and H1993 cells. ${ }^{* *} \mathrm{P}<0.01,{ }^{* * * *} \mathrm{P}<0.001$ and ${ }^{* * * * *} \mathrm{P}<0.0001$. MYG1, melanocyte proliferating gene 1; siRNA, small interfering RNA. 


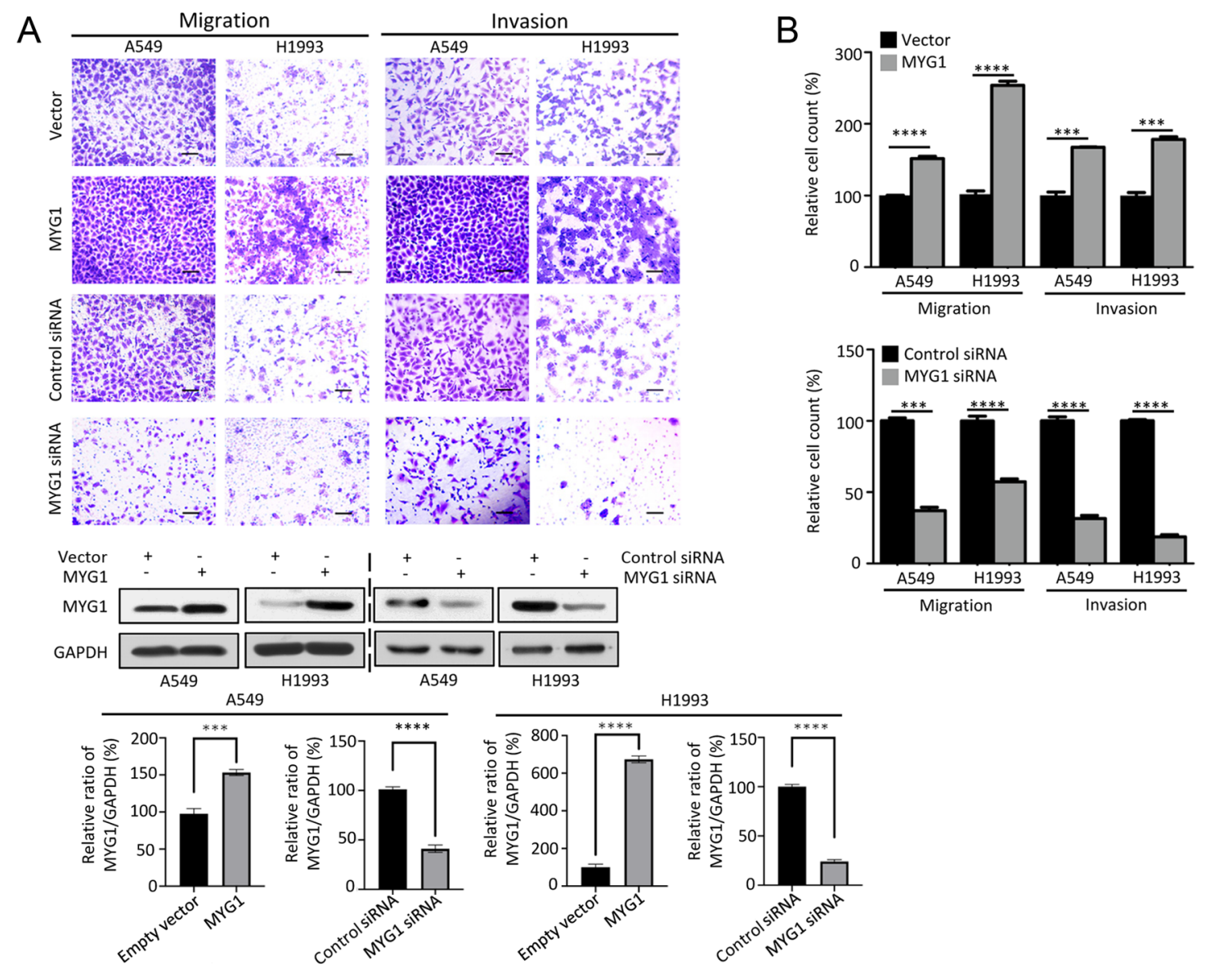

C
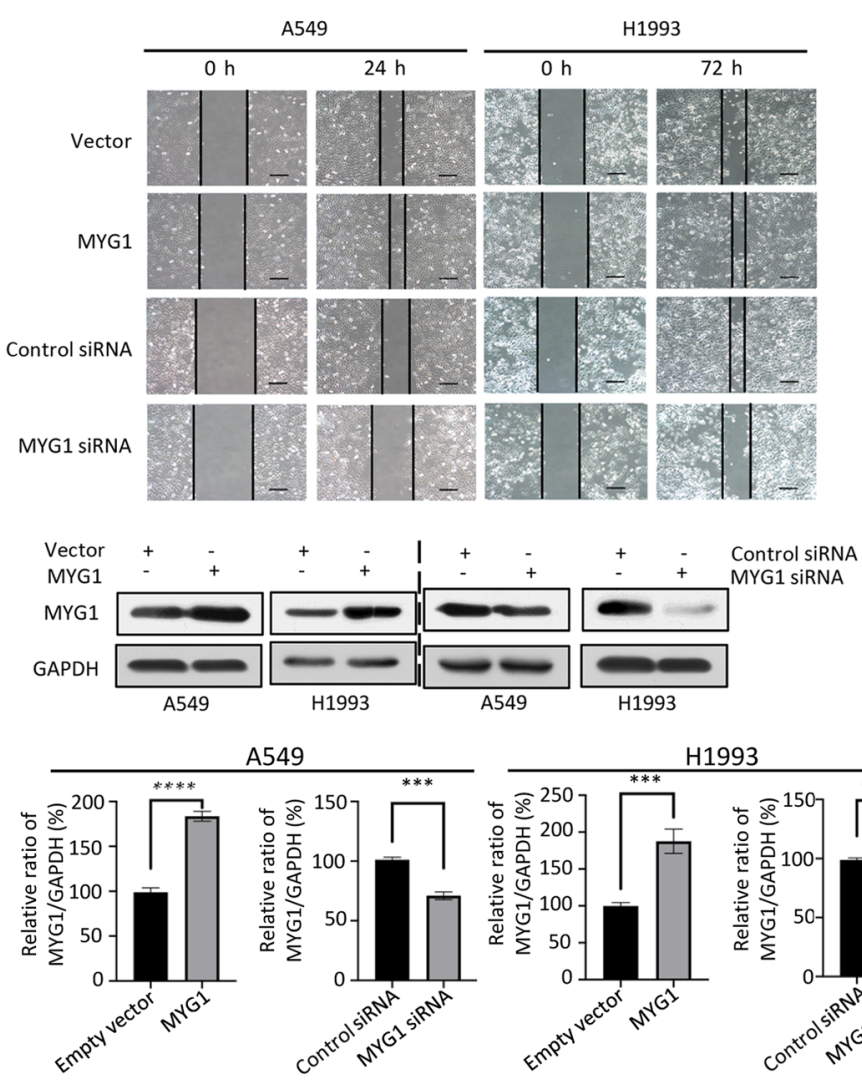

D
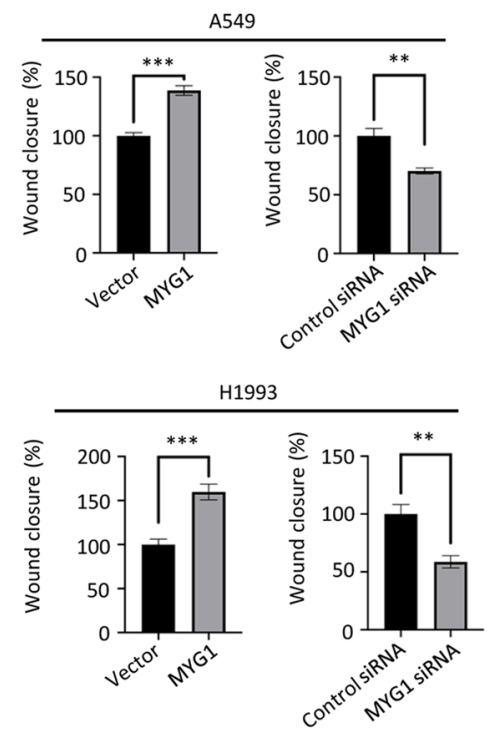

Figure 4. MYG1 promotes the migration and invasion of lung adenocarcinoma cells. (A) The results of the Transwell assays demonstrated that MYG1 promoted the migration and invasion of A549 and H1993 cells. Scale bar, $100 \mu \mathrm{m}$. (B) Quantitative results of the migration and invasion assays. (C) The results of the wound healing assay demonstrated that MYG1 promoted the migration of A549 and H1993 cells. Scale bar, $200 \mu \mathrm{m}$. (D) Quantitative results of the wound healing assay. ${ }^{* *} \mathrm{P}<0.01,{ }^{* * *} \mathrm{P}<0.001$ and ${ }^{* * * *} \mathrm{P}<0.0001$. MYG1, melanocyte proliferating gene 1 ; siRNA, small interfering RNA. 

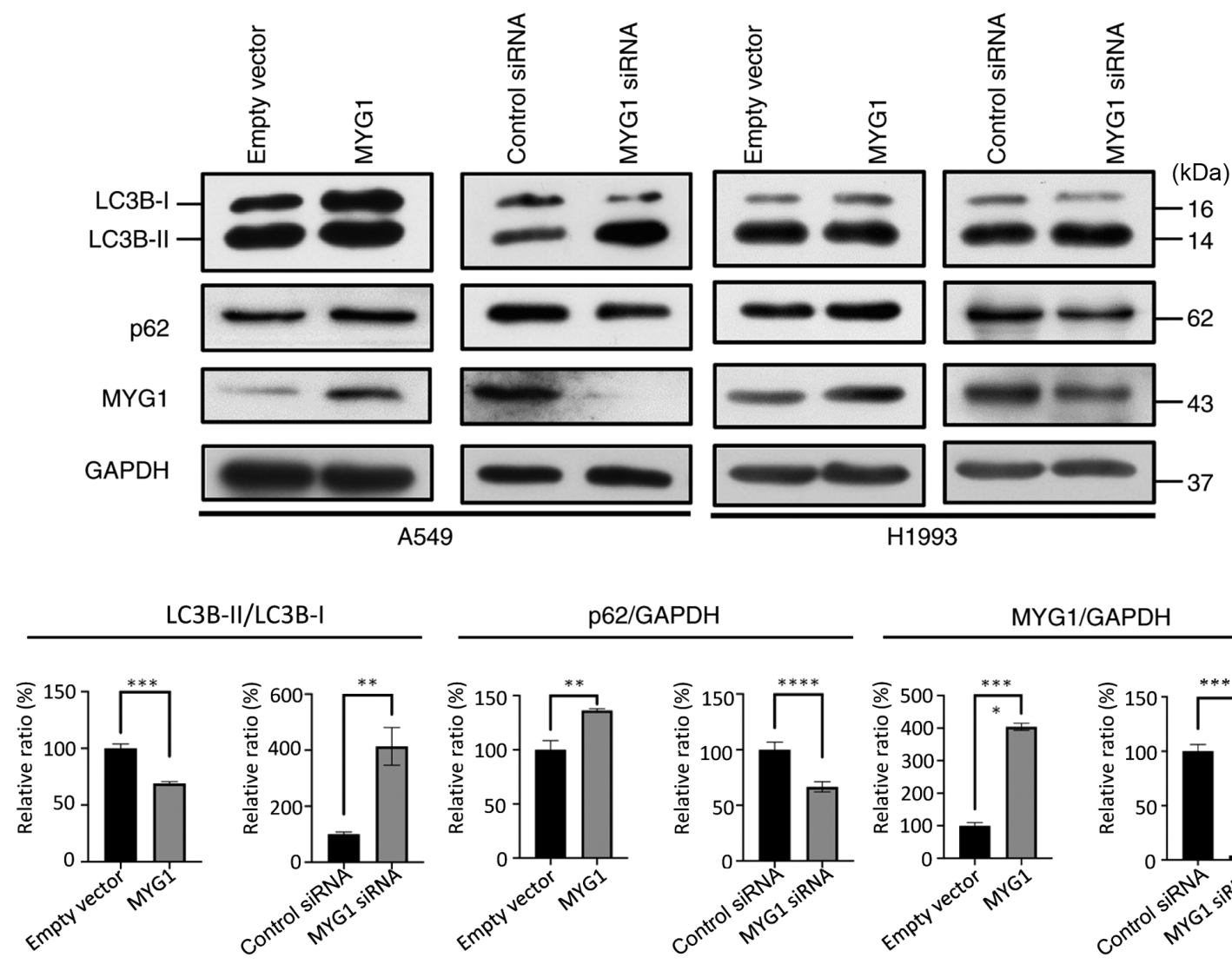

62/GAPDH

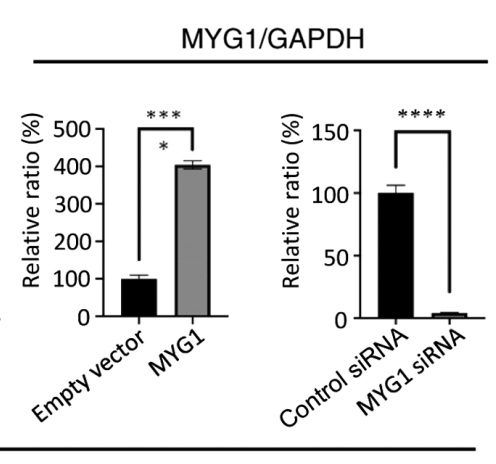

A549

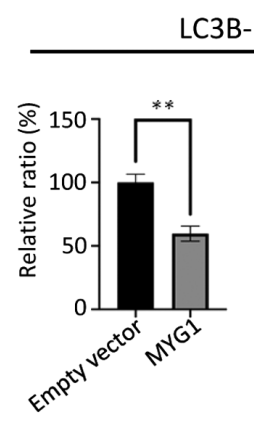

LC3B-II/LC3B-I
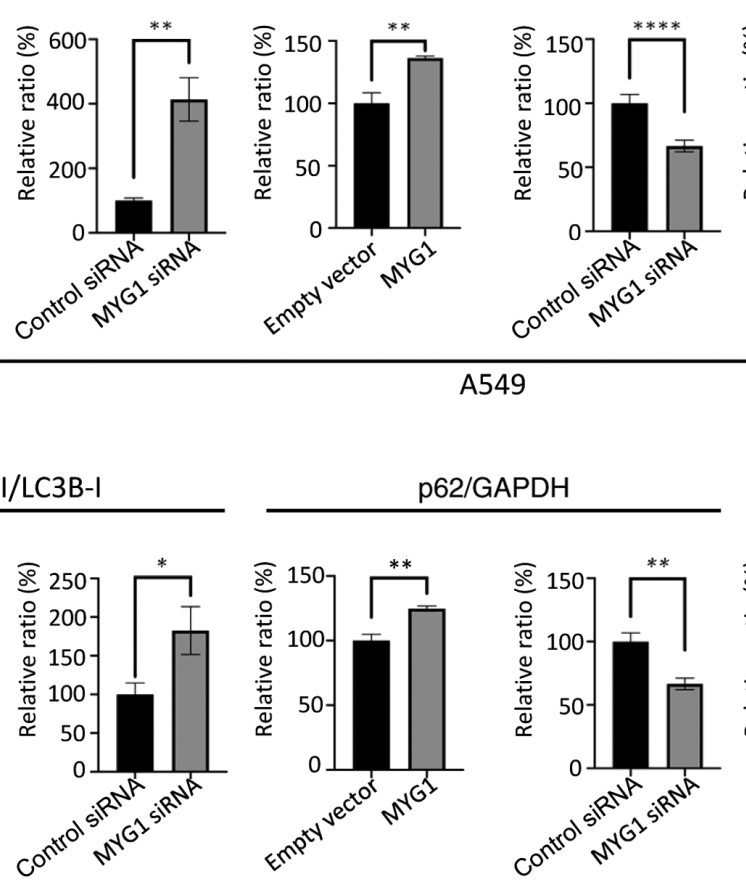

MYG1/GAPDH
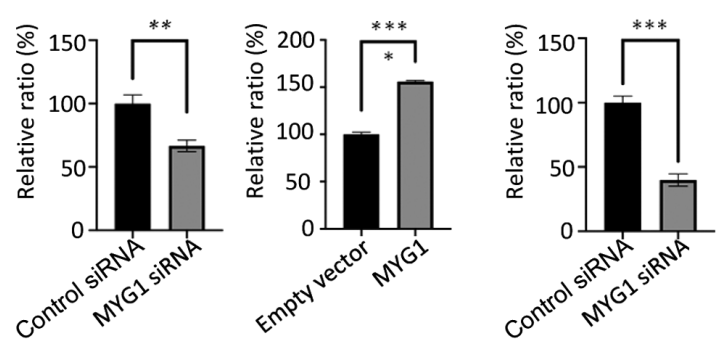

H1993

Figure 5. MYG1 inhibits autophagy in lung adenocarcinoma cells. The results of the western blot analysis demonstrated that overexpression of MYG1 suppressed the conversion of LC3-I to LC3-II and the degradation of p62 in A549 and H1993 cells compared with those in the empty vector-transfected cells. By contrast, knockdown of MYG1 enhanced the conversion of LC3-I to LC3-II and the degradation of p62 compared with those in the control siRNA group. ${ }^{*} \mathrm{P}<0.05,{ }^{* *} \mathrm{P}<0.01,{ }^{* * * *} \mathrm{P}<0.001$ and ${ }^{* * * * *} \mathrm{P}<0.0001$. MYG1, melanocyte proliferating gene 1; siRNA, small interfering RNA.

of LUAD. Subsequently, the association between the expression of MYG1 and the prognosis as well as the clinicopathological characteristics of patients with LUAD was evaluated, and the results indicated that high expression levels of MYG1 were associated with the $\mathrm{N}$ stage. Univariate analysis identified that high expression levels of MYG1 were associated with unfavorable OS and PPS rates. However, the results of the multivariate analysis indicated that MYG1 expression was not an independent indicator for either OS or PPS. Finally, in vitro experiments were performed to determine how high expression levels of MYG1 were associated with the progression of LUAD at the cellular level. The results demonstrated that MYG1 promoted the proliferation, migration and invasion, but suppressed autophagy.
As MYG1 knockout in yeast results in defects in respiratory growth due to its key role in processing ribosomal and messenger RNA transcripts in the mitochondria (8), we hypothesized that changes in MYG1 expression levels may affect the respiratory function of LUAD cells. The results of the GSEA on LUAD gene expression data suggested that high expression of MYG1 was enriched with the gene set of 'HALLMARK_ OXIDATIVE_PHOSPHORYLATION', which suggested that the change in MYG1 expression may affect the production of ATP, which is a product of oxidative phosphorylation. Thus, the amount of ATP was measured following MYG1 knockdown, and the results demonstrated the levels of ATP were reduced by $\sim 40 \%$ in the MYG1 knockdown group compared with those 
A

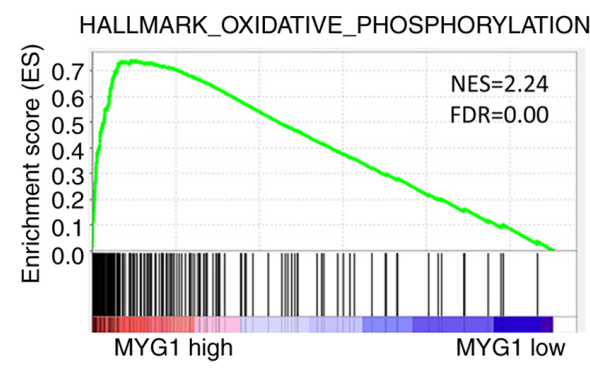

C

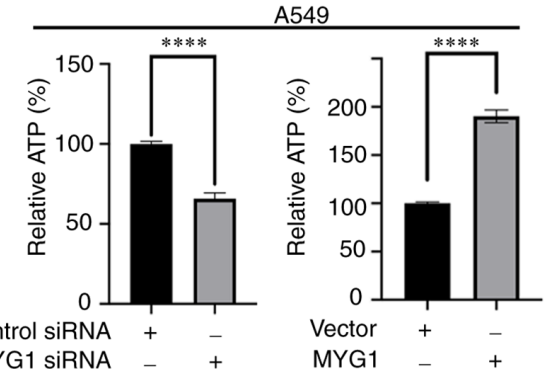

B
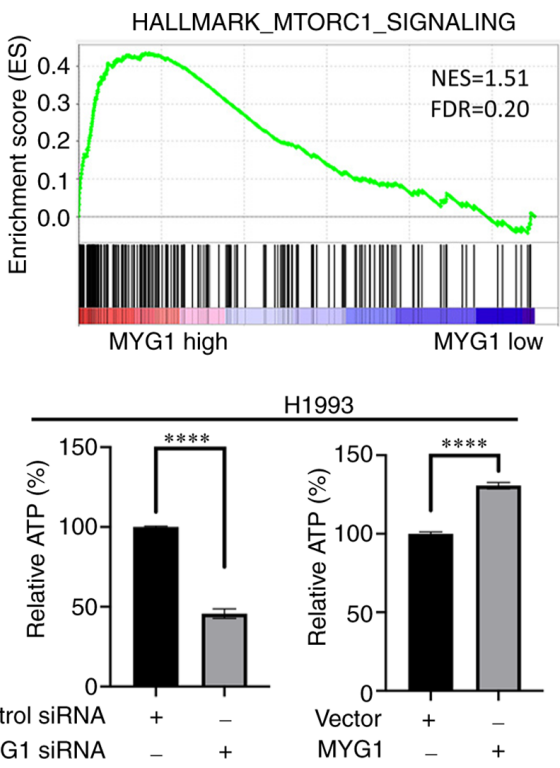

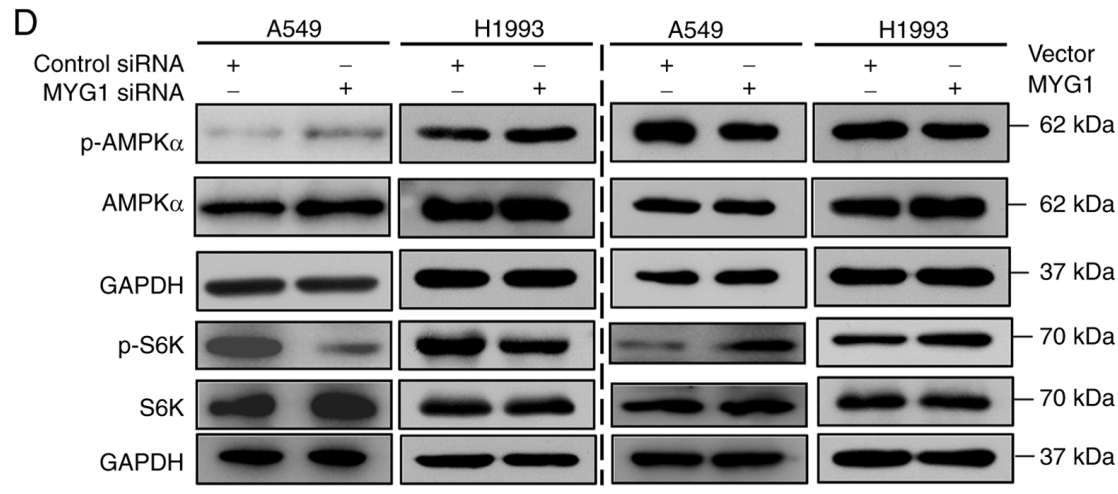

p-AMPK $\alpha / A M P K \alpha$
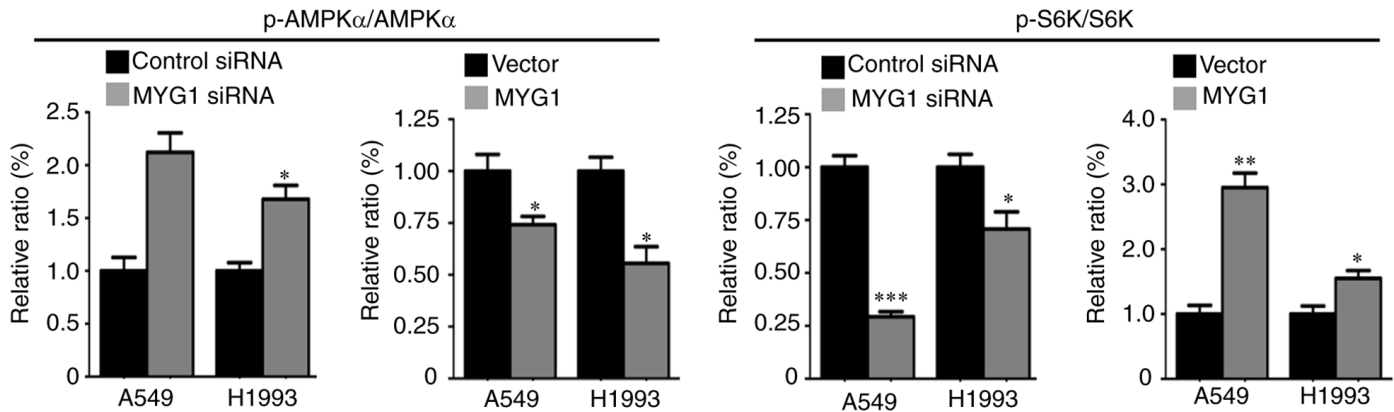

Figure 6. MYG1 positively regulates the AMPK/mTORC1 signaling pathway by promoting the production of ATP in lung adenocarcinoma cells. (A and B) The Gene Set Enrichment Analysis results indicated that the gene sets of 'HALLMARK_OXIDATIVE_PHOSPHORYLATION' and 'HALLMARK_MTORC1_ SIGNALING' were enriched with high expression of MYG1. (C) Knockdown of MYG1 suppressed the production of ATP in A549 and H1993 cells, whereas overexpression of MYG1 enhanced the production of ATP compared with that in the corresponding control groups. (D) Knockdown of MYG1 promoted the levels of phosphorylation of AMPK $\alpha$, but inhibited the levels of phosphorylation of p70S6K compared with those in the control siRNA-transfected group; overexpression of MYG1 exerted the opposite effects on the phosphorylation of AMPK $\alpha$ and p70S6K. $\mathrm{P}<0.05,{ }^{* *} \mathrm{P}<0.01,{ }^{* * * *} \mathrm{P}<0.001$ and ${ }^{* * * * *} \mathrm{P}<0.0001$. NES, normalized enrichment score; FDR, false discovery rate; MYG1, melanocyte proliferating gene 1; siRNA, small interfering RNA; AMPK $\alpha$, AMP-activated protein kinase $\alpha$; mTORC1, mTOR complex 1; p-, phosphorylated.

in the control group. As AMPK is a cellular energy sensor that is activated upon ATP depletion (5), and based on the GSEA results that suggested that the gene set of the $\mathrm{mTORC1}$ signaling pathway was enriched with high expression of MYG1, the activities of AMPK and mTORC1 were examined following MYG1 knockdown. The results demonstrated that knockdown of MYG1 increased the phosphorylation levels of AMPK $\alpha$ and inhibited mTORC1 activity, which was demonstrated by the decreased phosphorylation of S6K. mTORC1 serves a central role in regulating numerous cellular processes, including protein synthesis, lipid, nucleotide and glucose metabolism, and autophagy (4). Therefore, high expression levels of MYG1 may promote the cancerous features of LUAD cells and may be associated with an unfavorable prognosis. To the best of our knowledge, the present study was the first to reveal the role and mechanism of MYG1 in tumorigenesis. 
Since high MYG1 expression was observed in LUAD tissues and may contribute to cancerous features in the present study, the regulation of MYG1 expression LUAD should be further investigated. As the in vitro experiments in the present study were only performed in A549 and H1993 cells, further experiments should be performed in additional LUAD cell lines in order to confirm the present conclusions. In addition, since MYG1 expression was only accessed in TCGA LUAD tissue samples in the present study, its expression needs to be evaluated in other datasets to confirm the findings of this study. As it was observed that AJCC stage $\mathrm{N}$ was not an independent prognostic factor for OS, FP and PPS, and T stage was not an independent factor for PPS, these results may represent a potential limitation due to the cohort size. Therefore, more data from patients with LUAD should be analyzed to overcome this limitation. In addition, the expression levels and prognostic role of MYG1 in other types of cancer should be investigated.

In conclusion, the results of the present study demonstrated that the expression levels of MYG1 were upregulated in LUAD compared with those in normal lung tissues, and high expression of MYG1 was associated with unfavorable clinical outcomes. Furthermore, MYG1 may promote the proliferation, migration and invasion, as well as inhibit the autophagy of LUAD cells via the AMPK/mTORC1 signaling pathway. Collectively, the present results indicated that MYG1 may serve an oncogenic role in LUAD and may be a potential therapeutic target for LUAD.

\section{Acknowledgements}

Not applicable.

\section{Funding}

This work was supported in part by the Research Enhancement Project for Junior Faculty in Higher Education Institutes of Guangxi (grant no. 2019K Y0522), the Scientific Research Project for Junior Faculty in Guilin Medical College (grant no. 2018glmcy055), the Natural Science Foundation of Guangxi (grant no. 2020JJA140139) and the Open Research Fund from Guangxi Key Laboratory of Liver Injury and Repair Molecular Medicine (grant no. GXLIRMMKL-201802, GXLIRMMKL-201816). GH was supported by the Hundred Talents Program of Guangxi.

\section{Availability of data and materials}

The datasets used and/or analyzed during the current study are available from the corresponding author on reasonable request.

\section{Authors' contributions}

GH conceived the study and performed the bioinformatical analysis. XH, WW, CW, LD and AL performed the experiments. GH and $\mathrm{XH}$ confirm the authenticity of all the raw data. GH and XH analyzed and interpreted the data, and wrote the manuscript. All authors read and approved the final manuscript.

\section{Ethics approval and consent to participate}

Not applicable.

\section{Patient consent for publication}

Not applicable.

\section{Competing interests}

The authors declare that they have no competing interests.

\section{References}

1. Bray F, Ferlay J, Soerjomataram I, Siegel RL, Torre LA and Jemal A: Global cancer statistics 2018: GLOBOCAN estimates of incidence and mortality worldwide for 36 cancers in 185 countries. CA Cancer J Clin 68: 394-424, 2018.

2. Zappa $C$ and Mousa SA: Non-small cell lung cancer: Current treatment and future advances. Transl Lung Cancer Res 5: 288-300, 2016.

3. Herbst RS, Morgensztern D and Boshoff C: The biology and management of non-small cell lung cancer. Nature 553: 446-454, 2018.

4. Saxton RA and Sabatini DM: mTOR signaling in growth, metabolism, and disease. Cell 168: 960-976, 2017.

5. Herzig S and Shaw RJ: AMPK: Guardian of metabolism and mitochondrial homeostasis. Nat Rev Mol Cell Biol 19: 121-135, 2018.

6. Philips M-A, Vikeså J, Luuk H, Jønson L, Lilleväli K, Rehfeld JF, Vasar E, Kõks S and Nielsen FC: Characterization of MYG1 gene and protein: Subcellular distribution and function. Biol Cell 101: 361-377, 2009.

7. Philips MA, Kingo K, Karelson M, Rätsep R, Aunin E, Reimann E, Reemann P, Porosaar O, Vikeså J, Nielsen FC, et al: Promoter polymorphism-119C/G in MYG1 (C12orf10) gene is related to vitiligo susceptibility and Arg4Gln affects mitochondrial entrance of Myg1. BMC Med Genet 11: 56 , 2010.

8. Grover R, Burse SA, Shankrit S, Aggarwal A, Kirty K, Narta K, Srivastav R, Ray AK, Malik G, Vats A, et al: Mygl exonuclease couples the nuclear and mitochondrial translational programs through RNA processing. Nucleic Acids Res 47: 5852-5866, 2019.

9. Wang R, Wang W, Li A, Wang Y, Jin J, Huang Z and Huang G: Lipopolysaccharide enhances DNA-induced IFN- $\beta$ expression and autophagy by upregulating cGAS expression in A549 cells. Exp Ther Med 18: 4157-4164, 2019.

10. Grada A, Otero-Vinas M, Prieto-Castrillo F, Obagi Z and Falanga V: Research techniques made simple: Analysis of collective cell migration using the wound healing assay. J Invest Dermatol 137: e11-e16, 2017.

11. Zhang X, Zuo X, Yang B, Li Z, Xue Y, Zhou Y, Huang J, Zhao X, Zhou J, Yan Y, et al: MicroRNA directly enhances mitochondrial translation during muscle differentiation. Cell 158: 607-619, 2014.

12. Chandrashekar DS, Bashel B, Balasubramanya SAH, Creighton CJ, Ponce-Rodriguez I, Chakravarthi BVSK and Varambally S: UALCAN: A portal for facilitating tumor subgroup gene expression and survival analyses. Neoplasia 19: 649-658, 2017.

13. Győrffy B, Surowiak P, Budczies J and Lánczky A: Online survival analysis software to assess the prognostic value of biomarkers using transcriptomic data in non-small-cell lung cancer. PLoS One 8: e82241, 2013.

14. Mootha VK, Lindgren CM, Eriksson KF, Subramanian A, Sihag S, Lehar J, Puigserver P, Carlsson E, Ridderstråle M, Laurila E, et al: PGC-1alpha-responsive genes involved in oxidative phosphorylation are coordinately downregulated in human diabetes. Nat Genet 34: 267-273, 2003.

15. Subramanian A, Tamayo P, Mootha VK, Mukherjee S, Ebert BL, Gillette MA, Paulovich A, Pomeroy SL, Golub TR, Lander ES and Mesirov JP: Gene set enrichment analysis: A knowledge-based approach for interpreting genome-wide expression profiles. Proc Natl Acad Sci USA 102: 15545-15550, 2005. 
16. Hoadley KA, Yau C, Hinoue T, Wolf DM, Lazar AJ, Drill E, Shen R, Taylor AM, Cherniack AD, Thorsson V, et al: Cell-of-origin patterns dominate the molecular classification of 10,000 tumors from 33 types of cancer. Cell 173: 291-304.e6, 2018.

17. White E: The role for autophagy in cancer. J Clin Invest 125 : 42-46. 2015.

18. Klionsky DJ, Abdelmohsen K, Abe A, Abedin MJ, Abeliovich H, Acevedo Arozena A, Adachi H, Adams CM, Adams PD, Adeli K, et al: Guidelines for the use and interpretation of assays for monitoring autophagy (3rd edition). Autophagy 12: 1-222, 2016.
19. Vyas S, Zaganjor E and Haigis MC: Mitochondria and cancer. Cell 166: 555-566, 2016.

This work is licensed under a Creative Commons Attribution-NonCommercial-NoDerivatives 4.0 International (CC BY-NC-ND 4.0) License. 